\title{
Hardy-type inequalities on a half-space in the Heisenberg group
}

Heng-Xing Liu* and Jing-Wen Luan

\section{"Correspondence:}

hxliu.math@whu.edu.cn

School of Mathematics and

Statistics, Wuhan University, Wuhan,

430072, People's Republic of China

\section{Abstract}

We prove some Hardy-type inequalities on half-spaces for Kohn's sub-Laplacian in the Heisenberg group. Furthermore, the constants we obtained are sharp.

MSC: Primary 26D10; 35H20

Keywords: Hardy inequality; Heisenberg group; sharp constant

\section{Introduction}

The Hardy inequality in $\mathbb{R}^{N}$ reads that for all $u \in C_{0}^{\infty}\left(\mathbb{R}^{N}\right)$ and $N \geq 3$,

$$
\int_{\mathbb{R}^{N}}|\nabla u|^{2} d x \geq \frac{(N-2)^{2}}{4} \int_{\mathbb{R}^{N}} \frac{u^{2}}{|x|^{2}} d x
$$

and the constant $\frac{(N-2)^{2}}{4}$ in (1.1) is sharp. Recently, it has been proved by Nazarov ([1], Proposition 4.1, see also [2]) that the following Hardy inequality is valid for $f \in C_{0}^{\infty}\left(\mathbb{R}_{+}^{N}\right)$ :

$$
\int_{\mathbb{R}_{+}^{N}}|\nabla u(x)|^{2} d x \geq \frac{N^{2}}{4} \int_{\mathbb{R}_{+}^{N}} \frac{u(x)^{2}}{|x|^{2}} d x
$$

where $\mathbb{R}_{+}^{N}=\left\{\left(x_{1}, \ldots, x_{n}\right) \mid x_{1}>0\right\}$, and the constant $\frac{N^{2}}{4}$ is sharp. This shows that the Hardy constant jumps from $\frac{(N-2)^{2}}{4}$ to $\frac{N^{2}}{4}$ when the singularity of the potential reaches the boundary. For more information about this inequality and its applications, we refer to [3-10] and the references therein.

The aim of this note is to prove an analogous Hardy-type inequality on a half-space for Kohn's sub-Laplacian in Heisenberg groups $\mathbb{H}^{n}$. It has been proved by D'Ambrosio ([11], Theorem 3.3) that for $u \in C_{0}^{\infty}\left(\mathbb{H}^{n}\right)$, the following holds:

$$
\int_{\mathbb{H}^{n}}\left|\nabla_{H} u\right|^{2} d x d t \geq(n-1)^{2} \int_{\mathbb{H}^{n}} \frac{u^{2}}{|x|^{2}} d x d t
$$

where $\nabla_{H}$ is the horizontal gradient associated with Kohn's sub-Laplacian on $\mathbb{H}^{n}$ (for details, see Section 2). Furthermore, the constant $(n-1)^{2}$ in (1.3) is sharp (see [12], Theorem 3.13). In this note we shall show that when the singularity is on the boundary, the Hardy constant also jumps. In fact, we have the following.

C 2013 Liu and Luan; licensee Springer. This is an Open Access article distributed under the terms of the Creative Commons Attribution License (http://creativecommons.org/licenses/by/2.0), which permits unrestricted use, distribution, and reproduction in any medium, provided the original work is properly cited. 
Theorem 1.1 For all $u \in C_{0}^{\infty}\left(\mathbb{H}_{+}^{n}\right)$, the following holds:

$$
\int_{\mathbb{H}_{+}^{n}}\left|\nabla_{H} u\right|^{2} d x d t \geq n^{2} \int_{\mathbb{H}_{+}^{n}} \frac{u^{2}}{|x|^{2}} d x d t
$$

where $\mathbb{H}_{+}^{n}=\left\{(x, t) \in \mathbb{H}^{n}: x_{1}>0\right\}$, and the constant $n^{2}$ in (1.4) is sharp.

In order to prove Theorem 1.1, we use a new technique which is different from that in $[1,2]$. In fact, it seems that the method used in $[1,2]$ cannot be applied to Kohn's subLaplacian.

With the same technique, we obtain the following sharp Hardy inequality on $\mathbb{H}_{k_{+}}^{n}=$ $\left\{(x, t) \in \mathbb{H}^{n}: x_{1}>0, \ldots, x_{k}>0\right\}$.

Theorem 1.2 Let $1 \leq k \leq 2 n$. For all $u \in C_{0}^{\infty}\left(\mathbb{H}_{k_{+}}^{n}\right)$, the following holds:

$$
\int_{\mathbb{H}_{k_{+}}^{n}}\left|\nabla_{H} u\right|^{2} d x d t \geq(n+k-1)^{2} \int_{\mathbb{H}_{k_{+}}^{n}} \frac{u^{2}}{|x|^{2}} d x d t
$$

Furthermore, the constant $(n+k-1)^{2}$ in (1.5) is sharp.

\section{Proofs}

Let $\mathbb{H}^{n}=\left(\mathbb{R}^{2 n} \times \mathbb{R}, \circ\right)$ be the $(2 n+1)$-dimensional Heisenberg group whose group structure is given by

$$
(x, t) \circ\left(x^{\prime}, t^{\prime}\right)=\left(x+x^{\prime}, t+t^{\prime}+2 \sum_{j=1}^{n}\left(x_{2 j}^{\prime} x_{2 j-1}-x_{2 j-1}^{\prime} x_{2 j}\right)\right) .
$$

The vector fields

$$
X_{2 j-1}=\frac{\partial}{\partial x_{2 j-1}}+2 x_{2 j} \frac{\partial}{\partial t}, \quad X_{2 j}=\frac{\partial}{\partial x_{2 j}}-2 x_{2 j-1} \frac{\partial}{\partial t}
$$

$(j=1, \ldots, n)$ are left invariant and generate the Lie algebra of $\mathbb{H}^{n}$. Kohn's sub-Laplace on $\mathbb{H}^{n}$ is

$$
\Delta_{H}=\sum_{j=1}^{2 n} X_{j}^{2}=\sum_{j=1}^{2 n} \frac{\partial^{2}}{\partial x_{j}^{2}}+4|x|^{2} \frac{\partial^{2}}{\partial t^{2}}+4 \sum_{j=1}^{n}\left(x_{2 j} \frac{\partial}{\partial x_{2 j-1}}-x_{2 j-1} \frac{\partial}{\partial x_{2 j}}\right) \frac{\partial}{\partial t}
$$

and the horizontal gradient is the $(2 n)$-dimensional vector given by

$$
\nabla_{H}=\left(X_{1}, \ldots, X_{2 n}\right)=\nabla_{x}+2 \Lambda x \frac{\partial}{\partial t}
$$

where $\nabla_{x}=\left(\frac{\partial}{\partial x_{1}}, \ldots, \frac{\partial}{\partial x_{2 n}}\right), \Lambda$ is a skew symmetric and orthogonal matrix given by

$$
\Lambda=\operatorname{diag}\left(J_{1}, \ldots, J_{n}\right), \quad J_{1}=\cdots=J_{n}=\left(\begin{array}{cc}
0 & 1 \\
-1 & 0
\end{array}\right)
$$


By the definition of $\nabla_{H}$, we have, for $\alpha \in \mathbb{R}$ and $|x| \neq 0$,

$$
\begin{aligned}
\Delta_{H}\left(x_{1}|x|^{\alpha}\right) & =\sum_{j=1}^{2 n} \frac{\partial^{2}}{\partial x_{j}^{2}}\left(x_{1}|x|^{\alpha}\right)=\sum_{j=2}^{2 n} \frac{\partial^{2}}{\partial x_{j}^{2}}\left(x_{1}|x|^{\alpha}\right)+\frac{\partial^{2}}{\partial x_{1}^{2}}\left(x_{1}|x|^{\alpha}\right) \\
& =x_{1} \sum_{j=2}^{2 n} \frac{\partial^{2}}{\partial x_{j}^{2}}|x|^{\alpha}+x_{1} \frac{\partial^{2}}{\partial x_{1}^{2}}|x|^{\alpha}+2 \frac{\partial|x|^{\alpha}}{\partial x_{1}} \\
& =x_{1} \sum_{j=1}^{2 n} \frac{\partial^{2}}{\partial x_{j}^{2}}|x|^{\alpha}+2 \frac{\partial|x|^{\alpha}}{\partial x_{1}} \\
& =\alpha(2 n+\alpha) x_{1}|x|^{\alpha-2} .
\end{aligned}
$$

Similarly,

$$
\begin{aligned}
\Delta_{H}\left(|x|^{\alpha} \prod_{i=1}^{k} x_{i}\right) & =\sum_{j=1}^{2 n} \frac{\partial^{2}}{\partial x_{j}^{2}}\left(|x|^{\alpha} \prod_{i=1}^{k} x_{i}\right) \\
& =\sum_{j=k+1}^{2 n} \frac{\partial^{2}}{\partial x_{j}^{2}}\left(|x|^{\alpha} \prod_{i=1}^{k} x_{i}\right)+\sum_{l=1}^{k} \frac{\partial^{2}}{\partial x_{l}^{2}}\left(|x|^{\alpha} \prod_{i=1}^{k} x_{i}\right) \\
& =\prod_{i=1}^{k} x_{i} \sum_{j=1}^{2 n} \frac{\partial^{2}}{\partial x_{j}^{2}}|x|^{\alpha}+2 k|x|^{\alpha-2} \prod_{i=1}^{k} x_{i} \\
& =\alpha(2 n+2 k+\alpha-2)|x|^{\alpha-2} \prod_{i=1}^{k} x_{i} .
\end{aligned}
$$

Proof of Theorem 1.1 Using the substitution $u=x_{1}|x|^{-n} f$, we get

$$
\begin{aligned}
\int_{\mathbb{H}_{+}^{n}}\left|\nabla_{H} u\right|^{2} & =\int_{\mathbb{H}_{+}^{n}}\left[\left|\nabla_{H}\left(x_{1}|x|^{-n}\right)\right|^{2} f^{2}+\left|\nabla_{H} f\right|^{2} \frac{x_{1}^{2}}{|x|^{2 n}}+\frac{\left\langle\nabla_{H}\left(x_{1}^{2}|x|^{-2 n}\right), \nabla_{H} f^{2}\right\rangle}{2}\right] \\
& \geq \int_{\mathbb{H}_{+}^{n}}\left(\left|\nabla_{H}\left(x_{1}|x|^{-n}\right)\right|^{2} f^{2}+\frac{\left\langle\nabla_{H}\left(x_{1}^{2}|x|^{-2 n}\right), \nabla_{H} f^{2}\right\rangle}{2}\right) \\
& =\int_{\mathbb{H}_{+}^{n}} f^{2}\left(\left|\nabla_{H}\left(x_{1}|x|^{-n}\right)\right|^{2}-\frac{1}{2} \Delta_{H}\left(x_{1}^{2}|x|^{-2 n}\right)\right) .
\end{aligned}
$$

Using the following identity, for $g \in C^{2}\left(\mathbb{H}^{n}\right)$,

$$
\frac{1}{2} \Delta_{H} g^{2}=\frac{1}{2} \sum_{j=1}^{2 n} X_{j}^{2} g^{2}=g \sum_{j=1}^{2 n} X_{j}^{2} g+\sum_{j=1}^{m}\left|X_{j} g\right|^{2}=g \Delta_{H} g+\left|\nabla_{H} g\right|^{2}
$$

we have, by (2.1),

$$
\begin{aligned}
\left|\nabla_{H}\left(x_{1}|x|^{-n}\right)\right|^{2}-\frac{1}{2} \Delta_{H}\left(x_{1}^{2}|x|^{-2 n}\right) & =-x_{1}|x|^{-n} \Delta_{H}\left(x_{1}|x|^{-n}\right) \\
& =-x_{1}|x|^{-n} \cdot(-n) \cdot n x_{1}|x|^{-n-2} \\
& =n^{2} x_{1}^{2}|x|^{-2 n-2} .
\end{aligned}
$$


Therefore,

$$
\begin{aligned}
\int_{\mathbb{H}_{+}^{n}}\left|\nabla_{H} u\right|^{2} d x d t & \geq \int_{\mathbb{H}_{+}^{n}} f^{2}\left(\left|\nabla_{H}\left(x_{1}|x|^{-n}\right)\right|^{2}-\frac{1}{2} \Delta_{H}\left(x_{1}^{2}|x|^{-2 n}\right)\right) d x d t \\
& =n^{2} \int_{\mathbb{H}_{+}^{n}} f^{2} x_{1}^{2}|x|^{-2 n-2} d x d t \\
& =n^{2} \int_{\mathbb{H}_{+}^{n}} \frac{u^{2}}{|x|^{2}} d x d t .
\end{aligned}
$$

Now we show the constant $n^{2}$ in (1.4) is sharp. Choosing

$$
g(x, t)=\phi(x) \omega(t)
$$

where $\phi \in C_{0}^{\infty}\left(\mathbb{R}_{+}^{2 n}\right)$ and $\omega \in C_{0}^{\infty}(\mathbb{R})$, we have

$$
\nabla_{H} g(x, t)=\nabla_{x} g(x, t)+2 \Lambda x \frac{\partial}{\partial t} g(x, t)=\omega(t) \nabla_{x} \phi(x)+2 \phi(x) \omega^{\prime}(t) \Lambda x .
$$

Therefore,

$$
\begin{aligned}
\left|\nabla_{H} g(x, t)\right|^{2} & =\left\langle\omega(t) \nabla_{x} \phi(x)+2 \phi(x) \omega^{\prime}(t) \Lambda x, \omega(t) \nabla_{x} \phi(x)+2 \phi(x) \omega^{\prime}(t) \Lambda x\right\rangle \\
& =\omega^{2}(t)\left|\nabla_{x} \phi\right|^{2}+4 \phi^{2}|x|^{2}\left|\omega^{\prime}(t)\right|^{2}+4 \omega(t) \omega^{\prime}(t) \phi\left\langle\Lambda x, \nabla_{x} \phi\right\rangle
\end{aligned}
$$

To get the last equation, we use the fact $|\Lambda x|^{2}=|x|^{2}$.

Since

$$
\int_{-\infty}^{+\infty} \omega(t) \omega^{\prime}(t) d t=\frac{1}{2} \int_{-\infty}^{+\infty} \frac{d \omega^{2}(t)}{d t} d t=0
$$

we have, by (2.5),

$$
\begin{aligned}
\frac{\int_{\mathbb{H}_{+}^{n}}\left|\nabla_{H} g(x, t)\right|^{2} d x d t}{\int_{\mathbb{H}_{+}^{n}} \frac{g^{2}}{|x|^{2}} d x d t} & =\frac{\int_{\mathbb{H}_{+}^{n}} \omega^{2}(t)\left|\nabla_{x} \phi\right|^{2} d x d t+4 \int_{\mathbb{H}_{+}^{n}} \phi^{2}|x|^{2}\left|\omega^{\prime}(t)\right|^{2} d x d t}{\int_{\mathbb{R}_{+}^{2 n}} \frac{\phi^{2}}{|x|^{2}} d x \cdot \int_{\mathbb{R}} \omega^{2} d t} \\
& =\frac{\int_{\mathbb{R}_{+}^{2 n}}\left|\nabla_{x} \phi\right|^{2} d x}{\int_{\mathbb{R}_{+}^{2 n}} \frac{\phi^{2}}{|x|^{2}} d x}+4 \frac{\int_{\mathbb{R}}\left|\omega^{\prime}(t)\right|^{2} d t}{\int_{\mathbb{R}} \omega^{2} d t} \cdot \frac{\int_{\mathbb{R}_{+}^{2 n}} \phi^{2}|x|^{2} d x}{\int_{\mathbb{R}_{+}^{2 n}} \frac{\phi^{2}}{|x|^{2}} d x} .
\end{aligned}
$$

Notice that

$$
\inf _{\omega \in C_{0}^{\infty}(\mathbb{R}) \backslash\{0\}} \frac{\int_{\mathbb{R}}\left|\omega^{\prime}(t)\right|^{2} d t}{\int_{\mathbb{R}} \omega^{2} d t}=0,
$$

we have

$$
\inf _{u \in C_{0}^{\infty}\left(\mathbb{H}_{+}^{n}\right) \backslash\{0\}} \frac{\int_{\mathbb{H}_{+}^{n}}\left|\nabla_{H} u\right|^{2} d x d t}{\int_{\mathbb{H}_{+}^{n}} \frac{u^{2}}{|x|^{2}} d x d t} \leq \inf _{\phi \in C_{0}^{\infty}\left(\mathbb{R}^{2 n}\right) \backslash\{0\}} \frac{\int_{\mathbb{R}_{+}^{2 n}}\left|\nabla_{x} \phi\right|^{2} d x}{\int_{\mathbb{R}_{+}^{2 n}} \frac{\phi^{2}}{|x|^{2}} d x}=n^{2}
$$

Here we use the sharp Hardy inequality (1.2). This completes the proof of Theorem 1.1. 
Proof of Theorem 1.2 The proof is similar to that of Theorem 1.1. Using the substitution $u=f|x|^{-n-k+1} \prod_{i=1}^{k} x_{i}$, we get

$$
\begin{aligned}
\int_{\mathbb{H}_{k_{+}}^{n}}\left|\nabla_{H} u\right|^{2}= & \int_{\mathbb{H}_{k_{+}}^{n}}\left[\left|\nabla_{H}\left(|x|^{-n-k+1} \prod_{i=1}^{k} x_{i}\right)\right|^{2} f^{2}+\left|\nabla_{H} f\right|^{2} \frac{\prod_{i=1}^{k} x_{i}^{2}}{|x|^{2 n}}\right. \\
& \left.+\frac{1}{2}\left\langle\nabla_{H}\left(|x|^{-2 n-2 k+2} \prod_{i=1}^{k} x_{i}^{2}\right), \nabla_{H} f^{2}\right\rangle\right] \\
\geq & \int_{\mathbb{H}_{k_{+}}^{n}}\left(\left|\nabla_{H}\left(|x|^{-n-k+1} \prod_{i=1}^{k} x_{i}\right)\right|^{2} f^{2}+\frac{1}{2}\left\langle\nabla_{H}\left(|x|^{-2 n-2 k+2} \prod_{i=1}^{k} x_{i}^{2}\right), \nabla_{H} f^{2}\right\rangle\right) \\
= & \int_{\mathbb{H}_{k_{+}}^{n}} f^{2}\left(\left|\nabla_{H}\left(|x|^{-n-k+1} \prod_{i=1}^{k} x_{i}\right)\right|^{2}-\frac{1}{2} \Delta_{H}\left(|x|^{-2 n-2 k+2} \prod_{i=1}^{k} x_{i}^{2}\right)\right) .
\end{aligned}
$$

Using the identities (2.3) and (2.2), we have

$$
\begin{aligned}
& \left|\nabla_{H}\left(|x|^{-n-k+1} \prod_{i=1}^{k} x_{i}\right)\right|^{2}-\frac{1}{2} \Delta_{H}\left(|x|^{-2 n-2 k+2} \prod_{i=1}^{k} x_{i}^{2}\right) \\
& \quad=-|x|^{-n-k+1} \prod_{i=1}^{k} x_{i} \cdot \Delta_{H}\left(|x|^{-n-k+1} \prod_{i=1}^{k} x_{i}\right) \\
& =(n+k-1)^{2}|x|^{-2 n-2 k} \prod_{i=1}^{k} x_{i}^{2} .
\end{aligned}
$$

Therefore,

$$
\begin{aligned}
\int_{\mathbb{H}_{k_{+}}^{n}}\left|\nabla_{H} u\right|^{2} & \geq \int_{\mathbb{H}_{k_{+}}^{n}} f^{2}\left(\left|\nabla_{H}\left(|x|^{-n-k+1} \prod_{i=1}^{k} x_{i}\right)\right|^{2}-\frac{1}{2} \Delta_{H}\left(|x|^{-2 n-2 k+2} \prod_{i=1}^{k} x_{i}^{2}\right)\right) \\
& =(n+k-1)^{2} \int_{\mathbb{H}_{k_{+}}^{n}} f^{2}|x|^{-2 n-2 k} \prod_{i=1}^{k} x_{i}^{2} \\
& =(n+k-1)^{2} \int_{\mathbb{H}_{k_{+}}^{n}} \frac{u^{2}}{|x|^{2}} .
\end{aligned}
$$

To see the constant $(n+k-1)^{2}$ in $(1.5)$ is sharp, we consider the function

$$
h(x, t)=\psi(x) \omega(t)
$$

where $\psi \in C_{0}^{\infty}\left(\mathbb{R}_{k_{+}}^{2 n}\right)$ and $\omega \in C_{0}^{\infty}(\mathbb{R})$. Here we denote by $\mathbb{R}_{k_{+}}^{2 n}=\left\{x \in \mathbb{R}^{2 n}: x_{1}>0, \ldots, x_{k}>0\right\}$.

Then

$$
\begin{aligned}
\left|\nabla_{H} h(x, t)\right|^{2} & =\left\langle\omega(t) \nabla_{x} \psi(x)+2 \psi(x) \omega^{\prime}(t) \Lambda x, \omega(t) \nabla_{x} \psi(x)+2 \psi(x) \omega^{\prime}(t) \Lambda x\right\rangle \\
& =\omega^{2}(t)\left|\nabla_{x} \psi\right|^{2}+4 \psi^{2}|x|^{2}\left|\omega^{\prime}(t)\right|^{2}+4 \omega(t) \omega^{\prime}(t) \psi\left\langle\Lambda x, \nabla_{x} \psi\right\rangle
\end{aligned}
$$


and

$$
\begin{aligned}
\int_{\mathbb{H}_{k_{+}}^{n}}\left|\nabla_{H} h(x, t)\right|^{2} d x d t= & \int_{\mathbb{H}_{k_{+}}^{n}}\left(\omega^{2}(t)\left|\nabla_{x} \psi\right|^{2}+4 \psi^{2}|x|^{2}\left|\omega^{\prime}(t)\right|^{2}\right) d x d t \\
& +4 \int_{\mathbb{R}_{k_{+}}^{2 n}} \psi\left\langle\Lambda x, \nabla_{x} \psi\right\rangle d x \cdot \int_{\mathbb{R}} \omega(t) \omega^{\prime}(t) d t \\
= & \int_{\mathbb{H}_{k_{+}}^{n}}\left(\omega^{2}(t)\left|\nabla_{x} \psi\right|^{2}+4 \psi^{2}|x|^{2}\left|\omega^{\prime}(t)\right|^{2}\right) d x d t \\
& +4 \int_{\mathbb{R}_{k_{+}}^{2 n}} \psi\left\langle\Lambda x, \nabla_{x} \psi\right\rangle d x \cdot \frac{1}{2} \int_{\mathbb{R}} d \omega^{2}(t) \\
= & \int_{\mathbb{H}_{k_{+}}^{n}}\left(\omega^{2}(t)\left|\nabla_{x} \psi\right|^{2}+4 \psi^{2}|x|^{2}\left|\omega^{\prime}(t)\right|^{2}\right) d x d t .
\end{aligned}
$$

Therefore,

$$
\begin{aligned}
& \frac{\int_{\mathbb{H}_{k_{+}}^{n}}\left|\nabla_{H} h(x, t)\right|^{2} d x d t}{\int_{\mathbb{H}_{k_{+}}^{n}} \frac{h^{2}}{|x|^{2}} d x d t}=\frac{\int_{\mathbb{H}_{k_{+}}^{n}} \omega^{2}(t)\left|\nabla_{x} \psi\right|^{2} d x d t+4 \int_{\mathbb{H}_{k_{+}}^{n}} \psi^{2}|x|^{2}\left|\omega^{\prime}(t)\right|^{2} d x d t}{\int_{\mathbb{R}_{k_{+}}^{2 n}} \frac{\psi^{2}}{|x|^{2}} d x \cdot \int_{\mathbb{R}} \omega^{2} d t} \\
& =\frac{\int_{\mathbb{R}_{+}^{2 n}}\left|\nabla_{x} \psi\right|^{2} d x}{\int_{\mathbb{R}_{k_{+}}^{2 n}} \frac{\psi^{2}}{|x|^{2}} d x}+4 \frac{\int_{\mathbb{R}}\left|\omega^{\prime}(t)\right|^{2} d t}{\int_{\mathbb{R}} \omega^{2} d t} \cdot \frac{\int_{\mathbb{R}_{k_{+}}^{2 n}} \psi^{2}|x|^{2} d x}{\int_{\mathbb{R}_{k_{+}}^{2 n}} \frac{\psi^{2}}{|x|^{2}} d x} .
\end{aligned}
$$

Thus, by (2.6),

$$
\begin{aligned}
\inf _{u \in C_{0}^{\infty}\left(\mathbb{H}_{k_{+}}^{n}\right) \backslash\{0\}} \frac{\int_{\mathbb{H}_{k_{+}}^{n}}\left|\nabla_{H} u\right|^{2} d x d t}{\int_{\mathbb{H}_{k_{+}}^{n}} \frac{u^{2}}{|x|^{2}} d x d t} & \leq \inf _{\psi \in C_{0}^{\infty}\left(\mathbb{R}^{2 n}\right) \backslash\{0\}} \frac{\int_{\mathbb{R}_{+}^{2 n}}\left|\nabla_{x} \psi\right|^{2} d x}{\int_{\mathbb{R}_{k_{+}}^{2 n}} \frac{\psi^{2}}{|x|^{2}} d x} \\
& =(n+k-1)^{2} .
\end{aligned}
$$

Here we use the sharp Hardy inequality ([9], Theorem 1.1)

$$
\int_{\mathbb{R}_{+}^{2 n}}|\nabla f|^{2} d x \geq(n+k-1)^{2} \int_{\mathbb{R}_{+}^{2 n}} \frac{f^{2}}{|x|^{2}} d x .
$$

The proof of Theorem 1.2 is therefore completed.

\section{Competing interests}

The authors declare that they have no competing interests.

\section{Authors' contributions}

All authors contributed equally and significantly in writing this article. All authors read and approved the final manuscript.

\section{Acknowledgements}

The first author is supported by the National Natural Science Foundation of China (No. 11171259) and the second author is supported by the National Natural Science Foundation of China (No. 11201346). 


\section{References}

1. Nazarov, Al: Hardy-Sobolev inequalities in a cone. J. Math. Sci. 132(4), 419-427 (2006)

2. Filippas, S, Tertikas, A, Tidblom, J: On the structure of Hardy-Sobolev-Maz'ya inequalities. J. Eur. Math. Soc. 11(6), 1165-1185 (2009)

3. Caldiroli, P, Musina, R: Stationary states for a two-dimensional singular Schrödinger equation. Boll. Unione Mat. Ital, B 4(3), 609-633 (2001)

4. Caldiroli, P, Musina, R: Rellich inequalities with weights. Calc. Var. Partial Differ. Equ. 45(1-2), 147-164 (2012). doi:10.1007/s00526-011-0454-3

5. Cazacu, C: On Hardy inequalities with singularities on the boundary. C. R. Math. Acad. Sci. Paris, Sér. I 349, 273-277 (2011)

6. Cazacu, C: Hardy inequalities with boundary singularities. arXiv:1009.0931

7. Fall, MM: On the Hardy-Poincaré inequality with boundary singularities. arXiv:1008.4785

8. Fall, MM, Musina, R: Hardy-Poincaré inequalities with boundary singularities. Proc. R. Soc. Edinb., Sect. A, Math. 142(4), 769-786 (2012)

9. Su, D, Yang, Q-H: On the best constants of Hardy inequality in $\mathbb{R}^{n-k} \times\left(\mathbb{R}_{+}\right)^{k}$ and related improvements. J. Math. Anal. Appl. 389, 48-53 (2012)

10. Yang, Q: Hardy type inequalities related to Carnot-Carathéodory distance on the Heisenberg group. Proc. Am. Math. Soc. 141(1), 351-362 (2013)

11. D'Ambrosio, L: Some Hardy inequalities on the Heisenberg group. Differ. Equ. 40, 552-564 (2004)

12. D'Ambrosio, L: Hardy-type inequalities related to degenerate elliptic differential operators. Ann. Sc. Norm. Super. Pisa, Cl. Sci. IV, 451-486 (2005)

doi:10.1186/1029-242X-2013-291

Cite this article as: Liu and Luan: Hardy-type inequalities on a half-space in the Heisenberg group. Journal of Inequalities and Applications 2013 2013:291.

\section{Submit your manuscript to a SpringerOpen ${ }^{\odot}$ journal and benefit from:}

- Convenient online submission

Rigorous peer review

- Immediate publication on acceptance

- Open access: articles freely available online

- High visibility within the field

- Retaining the copyright to your article 